\title{
UN ESTUDIO DE LA PALABRA QUE EN CANCIONES HISPANAS
}

\author{
Norma Corrales Martin \\ Maritza Bell Corrales
}

\begin{abstract}
RESUMEN
Este artículo describe un paradigma de relaciones de la palabra que, una de las palabras más usadas en español. Para nuestro análisis utilizamos 37 canciones populares caribeñas, debido a la riqueza y variedad que tienen estas canciones en el uso del lenguaje.

Palabras clave: "que”, categoría gramatical, constituyentes, cláusula relativa, pronombre relativo.
\end{abstract}

\begin{abstract}
This article describes a paradigm of uses of the word que, one of the most peripatetic words in Spanish. For our analysis we chose 37 popular Caribbean songs due to the richness and variety of Spanish reflected in these songs.

Key words: "que", grammatical category, constituency, relative clause, relative pronoun.
\end{abstract}

\section{Introducción}

El presente artículo intenta un paradigma de relaciones de que, una de las palabras más usadas y más útiles en el idioma español.

Para cuantificar y explicar los constituyentes que usan que, hemos estudiado treinta y siete canciones de Colombia, Cuba, Miami y Nueva York (ciudades hispanas), Panamá, Puerto Rico, República Dominicana y Venezuela, elegidas por su riqueza gramatical y semántica. Todas las canciones han sido trabajadas en clases de español como segunda lengua. Justificamos el uso de canciones para el análisis, primero, porque las canciones son muestra de la lengua oral; segundo, porque las limitaciones de ritmo y rima inducen la creatividad lingüística, aunque vemos en los compositores un alto respeto por la gramaticalidad; tercero,

Ph. D. Norma Corrales Martin. Ohio University, Athens, OH. Profesora de español, Temple University, Philadelphia, PA.

Ph. D. Maritza Bell-Corrales. University of Florida, Gainesville, FL. Profesora de español y lingüística, Georgia Southern University, Statesboro, GA. 
porque las ideas por comunicar son generalmente expresadas en oraciones cortas; y last but not least, porque el material está siempre accesible en la memoria.

La necesidad de esta investigación es triple: por un lado, la escasez de estudios sobre el uso paradigmático de que en textos orales; por otro, hemos venido notando, a través del estudio de la gramática en canciones (Corrales 2001), que muchos ejemplos de que no pueden ser entendidos usando las explicaciones desarrolladas hasta el momento y., finalmente, por la pobreza explicativa en los libros universitarios de texto de español como segunda lengua sobre el uso de que.

Para empezar de la base, al escribir este artículo tomamos la definición del Diccionario Larousse. Este define que como una categoría morfológica que puede actuar en tres funciones principales: (1) un pronombre relativo equivalente a el, la o lo cual o los o las cuales. Por ejemplo: el hombre que ves, las mujeres que te hablan. (2) Una conjunción, cuyo propósito es enlazar dos cláusulas. Por ejemplo: trabaja tanto que no se le ve. Y (3) una interjección. Por ejemplo: ¡Que cantidad de trabajo!

Que es definido como categoría morfológica, y por las palabras a las cuales equivale. Pero la esencia del movimiento gracioso de que a lo largo y ancho del español, no se deja atrapar por clasificaciones. Simplemente catalogar usos de que no devela las reglas del juego que permiten al interlocutor decir: ¿Que qué? La práctica tradicional de definir una palabra por su equivalente tampoco es muy productiva, especialmente si se quiere enseñar los usos de que a alguien que estudia gramática hispana. La verdad es que tantos ques son confusos... ¿qués...?

Presentamos una propuesta como paradigma para entender el que español, basada (1) en el conocimiento aportado por los grandes maestros del español americano, principalmente Andrés Bello y Rufino José Cuervo; (2) en una adaptación al español de la gramática de casos de Fillmore (1968); y (3) en una adaptación al español de la teoría de los constituyentes de Bloomfield (1933) y Wells (1958).

Nuestra tesis esencial es que lo nominal es a lo morfológico como lo verbal es a lo sintáctico, es decir, dentro del constituyente las palabras tienen funciones morfológicas, nombre, preposición, enlace; en relación con el verbo, el eje del sistema sintáctico- semántico, los constituyentes establecen relaciones de caso con él. Así, estudiamos el uso de que, no solo dentro del constituyente, sino en su relación de caso con el verbo (Ver 2).

\section{Revisión de literatura}

El estudio de que en la literatura se relaciona con el devenir del pensamiento lingüístico. Diferentes escuelas se aproximan al uso y explicación de que de diversa manera.

\subsection{Criterio tradicional}

Según Bello (1847), la cláusula relativa se caracteriza como una construcción de dos oraciones relacionadas con un nexo relativo. El nexo de uso más frecuente, tanto en la lengua oral como en la lengua escrita, es que. Bello agrega que que puede ser sujeto, término de preposición o complemento del sustantivo reproducido, el cual se conoce como su antecedente. El antecedente, aunque se entiende como función de la cláusula relativa, no es constituyente de esta sino de la oración principal.

Siguiendo esta modalidad tradicional, las cláusulas de relativo se clasifican en especificativas o restrictivas y explicativas. Las cláusulas especificativas determinan el antecedente restringiendo su campo de significación. Por ejemplo: el libro que está en la 
mesa es interesante, especifica cuál es el libro interesante; por lo tanto, no se puede prescindir de la oración subordinada. Las cláusulas explicativas, por otro lado, explican una cualidad o circunstancia del antecedente. Por ejemplo: Pedro, que es mi vecino, está enfermo. En este ejemplo, lo importante es el hecho de que Pedro está enfermo y, si se suprime la oración subordinada, no se altera el significado de la principal.

Por otro lado, Gil (2000) distingue cuatro tipos de que en la gramática de Bello: el que sustantivado, el que anunciativo, el que con artículo sustantivado, el que con artículo no sustantivado.

Nuessel (1975) distingue entre el que que introduce una oración subordinada, el que anunciativo, que 'no desempeña ninguna función en la oración que introduce', y el que con fuerza ilocucionaria.

La frecuencia de uso de que ha sido confirmada por Cortés Rodríguez (1987), quien señala que el porcentaje de aparición de que en relación con el resto de los relativos es de un 95 por ciento, y George DeMello (1993), quien, en su estudio sobre pronombres relativos con antecedente humano en varias ciudades de Suramérica y España, encontró que que sobrepasa a quien con un 53 por ciento de la totalidad de los casos investigados.

\subsection{Criterio estructuralista}

Según E. Alarcos (1973), los pronombres relativos tienen el oficio de transpositores del núcleo verbal a la función adjetiva, mientras que los adyacentes nominales (conjunciones subordinantes) sirven de transpositores del núcleo verbal a la función nominal. En este caso, se hablaría de oraciones subordinadas sustantivas. Por ejemplo: Anunció sus propósitos = Anunció que vendría.

De acuerdo con Alarcos, el transpositor que puede convertir la oración originaria en un segmento que funciona como los adjetivos y señala determinadas funciones cumplidas ordinariamente por sintagmas nominales a los que hace referencia. Por ejemplo: la lluvia que caía (función sujeto), la fruta que has comido (función complemento directo). Alarcos considera que no hay criterios gramaticales que puedan justificar la distinción de tipos de cláusula relativa. La distinción impartida por las pausas que se hacen en las explicativas y la falta de pausa en las restrictivas no constituye una distinción gramatical sino morfológica.

También de acuerdo con el análisis estructural, Pruñosa-Tomás (1990) describe la cláusula relativa como una construcción por la cual una oración pasa a ser parte integral en el esquema de relaciones estructurales de otra oración, con la función de término adyacente de un elemento nominal de la oración principal.

Alonso (1991) usando ejemplos de literatura y habla, postula para que y otros pronombres relativos el valor de $q u e^{2}$, en cuanto transpositores y funtivos. Los relativos son transpositores de segundo grado en cuanto la categoría de partida o transferendo es un sintagma verbal. Los relativos son funtivos en cuanto, en contra de su dependencia, cumplen una función gramatical dentro de la oración transpuesta. Alonso está en desacuerdo con Alarcos (1981) y otros autores que identifican oraciones adjetivas con oraciones de relativo, desde que algunos relativos pueden ejercer transposiciones de oraciones a categoría sustantiva. Desprendiéndose del criterio tradicional, Alonso iguala oración a verbo 'los verbos, esto es, las oraciones...' (1991: 325), señala que el artículo actúa como transpositor a categoría sustantiva (1991: 332), y adhiere al concepto de que el adverbio es un nombre inmovilizado en género y número (1991: 332). 


\subsection{Criterio generativo-transformacional}

En cuanto a las cláusulas relativas, la tarea de la gramática generativa-transformacional consiste en determinar el esquema sintáctico adecuado para explicar el mecanismo subyacente en la actividad linguiística del hablante. La caracterización de la cláusula relativa se realiza primordialmente a partir de su aspecto sintáctico. Como hecho sintáctico se toma en cuenta la delimitación utilizada por las gramáticas tradicionales: se trata de una oración constituyente que forma parte de otra, denominada oración matriz u oración principal.

Dentro de los postulados de la nueva sintaxis tenemos la regla de desplazamiento de $q u$. Según María Luisa Rivero (1982), en su estudio de las relativas especificativas o restrictivas, un sintagma que contiene el morfema $\boldsymbol{q u}$ es trasladado a la posición inicial de cláusula por medio de una transformación de movimiento. Una regla de elisión suprime los sintagmas carentes de caso o un caso diferente del oblicuo. Los sintagmas relativos oblicuos, es decir, los que son constituyentes de un sintagma preposicional, no se eliden. Rivero añade que hay un filtro de superficie que hace obligatoria la presencia del complementante o conjunción que si el relativo ha sido eliminado.

Rivero niega que en cláusulas del tipo El profesor que visitamos, que sea un pronombre relativo, y afirma que es solo un complementante o conjunción; mientras que sí es un pronombre relativo cuando la cláusula está introducida por preposición. Indica que la gramática del español tiene un pronombre relativo que, homófono del complementante, y una secuencia relativa el que. Estos relativos aparecen como constituyentes de un sintagma preposicional. En El martillo con que clavo, que es un pronombre relativo y no se puede identificar con la conjunción. Sin embargo, los ejemplos que Rivero emplea para su análisis inicial de la estructura de las relativas especificativas, el profesor que habla, parecen contradecir su presupuesto.

Todos estos análisis, llámense tradicionales, estructurales, generativos o pragmáticos, resultan más o menos confusos porque los presupuestos en que se basan pertenecen aún a la gramática morfológica, cuyo eje es el nombre-sujeto. Vemos (1) cómo se trata de dar categorías morfológicas a conglomerados sintácticos ('en con los que venimos, que venimos es adjetivo, los que venimos es sustantivo, con los que venimos es adverbio'. Álvarez 1986: 119), sin tener en cuenta el contexto sintáctico-semántico en que se realizan: su relación de caso con el verbo principal $\left(\mathrm{v}_{1}\right)$; (2) se basa el análisis en el concepto de oración compuesta por sujeto y predicado, $\mathrm{O} \rightarrow \mathrm{SN}$ SV, criterio meramente morfológico, en donde el sujeto se pone al mismo nivel del verbo en la oración; y (3) hay confusión en el uso de términos como oración relativa, cláusula relativa, frase relativa y pronombre relativo.

\section{Marco teórico de la propuesta}

A continuación, presentamos nuestra propuesta de una gramática verbal, un proceso análitico para estudiar la lengua y, en este caso, que. Comparamos la lengua con el sistema solar: el verbo es el eje de la oración, es como el sol, alrededor del cual giran los planetas, los constituyentes, a veces con satélites, cada uno en una órbita diferente, los diferentes casos del verbo. En los ejemplos, el signo igual (=) indica equivalencia, el verbo siempre está subrayado y los paréntesis ( ) indican ya carácter tácito, ya opcional.

En el nivel semántico del discurso, cada acción mencionada va acompañada de otras palabras que informan el quién, qué, a quién, cómo, cuándo, dónde, etc., de la acción: 
(1)

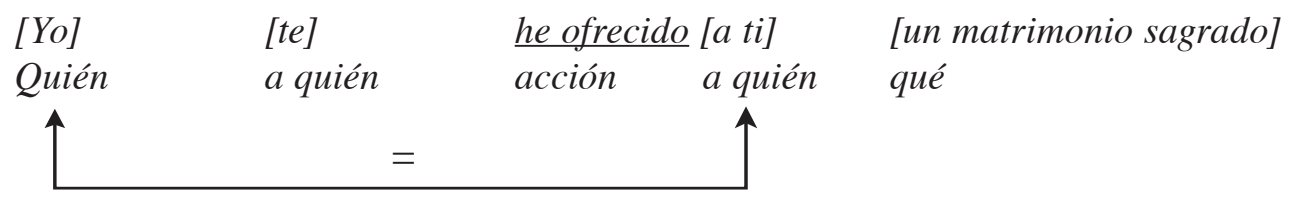

En el nivel sintáctico del discurso, cada verbo conjugado (simple, compuesto o perifrástico) va acompañado de nombres que establecen con él una determinada relación de caso, agente y paciente, persona y cosa, modo, tiempo, espacio, etc.; aunque siendo el verbo el centro de la oración, puede estar solo: Llueve.

\begin{tabular}{|c|c|c|c|c|}
\hline \multirow{4}{*}{$\begin{array}{l}\text { [Yo] } \\
\text { Agente }\end{array}$} & [te] & he ofrecido & \multicolumn{2}{|c|}{ [a ti] [un matrimonio sagrado] } \\
\hline & paciente & verbo & paciente & paciente \\
\hline & persona & & persona & $\cos a$ \\
\hline & 个 & $=$ & 个 & \\
\hline
\end{tabular}

Los verbos no conjugados o verbales también se acompañan de nombres en relación de caso con ellos:

\begin{tabular}{|c|c|c|c|c|}
\hline$(Y o)$ & [Desde muy niño] & luché & [pa' conseguir & [la fama]] \\
\hline Agente & tiempo & verbo & finalidad & paciente \\
\hline
\end{tabular}

En el nivel morfológico del discurso, cada caso del verbo es un constituyente de la oración con un núcleo 'protonominal'. La oración es definida como:

$$
\mathrm{O}=\text { modalidad } \underline{\mathrm{v}}[\mathrm{n}]_{\mathrm{x}}
$$

donde la modalidad refleja la intención del hablante de afirmar, negar, interrogar, etc.; $\underline{\mathrm{v}}$ es el verbo conjugado, definido como $\mathrm{v}_{1}, \mathrm{v}_{2}$, etc.; $[\mathrm{n}]_{\mathrm{x}}$ es el número de constituyentes que establecen relación de caso con el verbo.

Los constituyentes funcionan como unidades de sentido y contienen desde el núcleo solo, [Yo] vengo, hasta la cláusula relativa, definida por la presencia de un verbo ${ }_{2}$ :

[[Las cartas] $=[q u e]$ escribí] [nunca] [las] envié.

En el ejemplo, el verbo principal $\left(\mathrm{v}_{1}\right)$ es envié; el agente de envié está tácito (yo); el nombre las cartas, es el núcleo del constituyente [[Las cartas] = [que] escribí]] y se relaciona con envié como paciente. Que tiene dentro del constituyente una función triple:

(a) como enlace, une el nombre y el verbo ${ }_{2}$;

(b) como pronombre relativo, reproduce al nombre cartas;

(c) como nombre, es caso paciente cosa del verbo 2 , escribí. Que no constituye relación de caso con el verbo . 
La cláusula relativa como constituyente de la oración puede ser expresada:

$\mathrm{O}=\mathrm{v}_{1} \quad[\mathrm{c}($ láusula) relativa $]$

donde,

$[\mathrm{c}$ relativa $]=\left[[\mathrm{n}]=[\right.$ que $\left.] \mathrm{v}_{2}\right]$

Sin embargo, no todo constituyente con que y un verbo ${ }_{2}$ es una cláusula relativa. En el siguiente ejemplo que tiene diferentes funciones que comparamos con las anotadas arriba:

(Yo) $\underline{\text { sé }}[[q u e]=[[t u ́]$ no podrás olvidar $]]$<smiles></smiles>

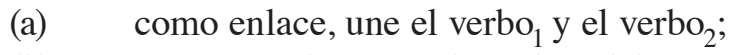

(b) como nombre, es el núcleo del constituyente, [[que] $=$ [ [tú] no podrás olvidarl], paciente cosa del verbo ${ }_{1}$, sé, '¿qué sé?'.

(c) dentro del constituyente que reproduce el verbo ${ }_{2}$ y posibles constituyentes: [no podrás olvidar] es 'que'. Que no establece relación de caso con el $\mathrm{v}_{2}$.

Dentro del constituyente este que desempeña una función morfológica diferente a la del que relativo. Que mantiene su carácter reproductivo, pero en este caso catafóricamente reproduce al verbo ${ }_{2}$. Esta función de que reproduciendo al verbo ${ }_{2}$ no está descrita de esta manera en la literatura estudiada. Proponemos el término 'proverbo' para nombrarla. La relación indicada puede ser expresada así:

$\mathrm{O}=\mathrm{v}_{1}$ [c(láusula) proverbal]

donde,

$[\mathrm{c}$ proverbal $]=\left[(\right.$ prep $)[$ que $]=\left[\left[\mathrm{v}_{2}\right]\right]$

La reproducción del verbo ${ }_{2}$ también sucede en presencia de una preposición (prep), en nuestro material comúnmente por o para.

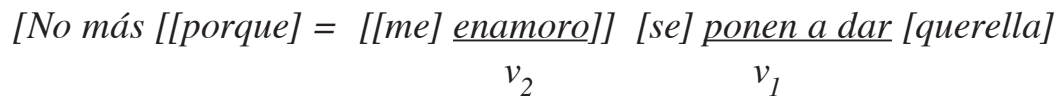

Que antecedido por la preposición por es el núcleo del constituyente razón del $\mathrm{v}_{1}$, o sea, la razón de que se pongan a dar querella es porque me enamoro; a su vez, me enamoro es el que del porque. Que reproduce al $\mathrm{v}_{2}$, enamoro sin establecer relación de caso con él.

En resumen, nuestro análisis se rige por los siguientes postulados:

El verbo es el componente fundamental y el eje de la oración (Fillmore 1968). La información semántica que el verbo conlleva, ¿quién, qué, a quién, cómo, cuándo, dónde, hizo?, se comunica a través de constituyentes morfológicos, a veces de alta complejidad. El núcleo del constituyente es siempre un nombre (sustantivo, adjetivo, adverbio), o pronombre, o el proverbo que. 
La cláusula relativa se caracteriza porque el núcleo del constituyente es un nombre o pronombre que se relaciona con el verbo 1 y por la presencia de un verbo ${ }_{2}$ introducido por que. La función de que en el constituyente es triple. Como enlace relaciona el nombre núcleo del constituyente con una acción que le caracteriza; como pronombre que reproduce al nombre y, como nombre, independientemente de su antecedente, constituye con el verbo ${ }_{2}$ relaciones de caso varias. Este que tiene un valor anafórico.

La cláusula proverbal se caracteriza porque el núcleo del constituyente es que en

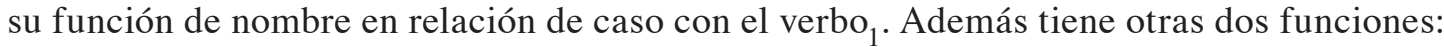

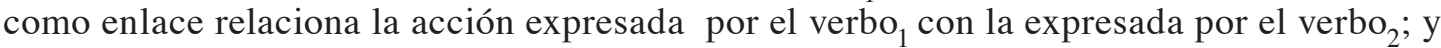
como proverbo reproduce al verbo ${ }_{2}$ sin establecer relación de caso con él. Tiene el carácter reproductivo del que relativo, pero en este caso reproduce al verbo ${ }_{2}$. Este que tiene un valor catafórico.

Sentada nuestra tesis esencial, otros usos de que en el material son:

- Qué pronombre interrogativo. En la interrogativa directa: ¿Qué dirán de mí? ¿Por qué no me has vuelto a llamar? ¿Qué número tú jugaste? En la interrogativa indirecta: Dime qué te han dicho.

- Que con $\emptyset$ verbo ${ }_{1}$ ó que pragmático, el cual se presenta así: qué exclamativo, iqué cosa más rica!, que declarativo, que nos pegamos, y que desiderativo, que el cielo te colme de habichuelas.

- Que usado como conjunción uniendo dos verbos en el nivel sintáctico: Dale que dale, o dos nombres en el nivel morfológico: [Lucero espiritual] eres [más alto $<$ que $>$ el hombre].

A continuación, aplicaremos los postulados descritos al conteo y explicación de los usos de que en el material estudiado.

\section{Presentación y análisis de datos}

En total que es usado 343 veces en las 37 canciones, bajo la siguiente distribución, siguiendo los parámetros descritos:

Tabla 1. Usos de que en las canciones

\begin{tabular}{c} 
Que 'proverbo' \\
$\mathrm{v}_{1}+\left[[\right.$ (preposición) que $\left.]=\left[\mathrm{v}_{2}\right]\right]$ \\
Que pronombre relativo \\
$\mathrm{v}_{1}+\left[[\right.$ nombre $]=[$ que $\left.] \mathrm{v}_{2}\right]$ \\
\hline continúa...
\end{tabular}


...continuación

Tabla 1. Usos de que en las canciones

\begin{tabular}{|c|c|}
\hline $\begin{array}{c}\text { Interrogativa directa } \\
{\left[(\text { prep) qué (nombre) }] \mathrm{v}_{1}\right.}\end{array}$ & 8 \\
\hline $\begin{array}{l}\text { Interrogativa indirecta } \\
\left.\left.\mathrm{v}_{1}+[] \text { (prep) qué }\right] \mathrm{v}_{2}\right]\end{array}$ & 8 \\
\hline $\begin{array}{c}\text { Que pragmático } \\
\emptyset_{\text {verbo }_{1}}[\text { qué nombre }] \\
\varnothing_{\text {verbo }_{1}}\left[\text { que }=\mathrm{v}_{2}\right]\end{array}$ & 39 \\
\hline Que conjunción & 12 \\
\hline Total & 343 \\
\hline
\end{tabular}

Usaremos las siguientes convenciones en el análisis:

Agente persona: $\quad$ agp

Agente cosa: agc

Agente reflexivo: agr

Experimentador: $\quad \exp$

Paciente persona: $\quad$ pp

Paciente cosa: $\quad$ pc

Instrumento: inst

Proverbo: $\quad$ pvb

Pronombre relativo: prr

Pronombre interrogativo: pi

Verbo 1: $\quad \mathrm{v}_{1}$

Verbo 2: $\quad \mathrm{v}_{2}$

Preposición: prep

4.1. Que 'proverbo', verbo $1[$ que $\left.]=\left[v_{2}\right]\right]$

Tabla 2. Que 'proverbo', verbo ${ }_{1}\left[[q u e]=\left[v_{2}\right]\right]$

\begin{tabular}{|c|c|c|c|c|c|c|}
\hline $\begin{array}{c}\text { Verbo } \\
\text { Caso del verbo }\end{array}$ & Decir & Saber & Ser/estar & Haber & Otros verbos & Total \\
\hline Agente persona & & & & & & 00 \\
\hline Agente cosa & 1 & & 12 & & 8 & 21 \\
\hline
\end{tabular}


Tabla 2. Que 'proverbo', verbo $1[$ que $\left.]=\left[\mathrm{v}_{2}\right]\right]$

\begin{tabular}{|c|c|c|c|c|c|c|}
\hline $\begin{array}{c}\text { Verbo } \\
\text { Caso del verbo }\end{array}$ & Decir & Saber & Ser/estar & Haber & Otros verbos & Total \\
\hline Experimentador* & & & & & & 0 \\
\hline Paciente persona & & & & & 0 & 0 \\
\hline Paciente cosa & 17 & 14 & & 4 & 27 & 62 \\
\hline Modal & & & 1 & & 1 & 2 \\
\hline Total & 18 & 14 & 13 & 4 & 36 & 885 \\
\hline
\end{tabular}

*Experimentador es el caso de El paraguas se me olvidó en la guagua y Los callos se me han caído, exclusivo de personas.

Encontramos 85 usos de que como enlace, nombre y 'proverbo' sin preposición (v. 3.2). En estos ejemplos, el nombre que es el núcleo del constituyente proverbal, relacionado con el verbo $_{1}$ en una determinada relación de caso, y al mismo tiempo que es 'proverbo' del verbo ${ }_{2}$, es decir, reproduce al verbo ${ }_{2}$, sin establecer relación de caso con él. Dividimos el material para reflejar los verbos con los que esto sucede más a menudo. El verbo más usado fue decir con 18 ejemplos. La relación de caso más común entre el verbo y el proverbo que es la de paciente cosa con 62 ejemplos. Le sigue el agente cosa con 21 ejemplos. No se encontraron casos en que la cláusula [que $=\mathrm{v}_{2}$ ] fuera el agente persona, experimentador o paciente persona del verbo ${ }_{1}$. Ejemplos selectos de estos casos siguen.

\subsubsection{Agente cosa}

El constituyente proverbal con que es agente cosa del $v_{1}$ en 21 ejemplos. De estos, 12 se producen con ser, 8 con verbos como sobrar, pasar, ocurrir, importar, parecer y olvidar, cuyo agente es típicamente no humano (agente cosa), y uno con decir. El agente de estos verbos es aquel constituyente susceptible de ser reemplazado por el pronombre agente neutro ello. Adherimos a la tesis de Bello (1964: 125) de que es 'un hecho manifiesto en que no cabe disputa' que el verbo ser rige acusativo.

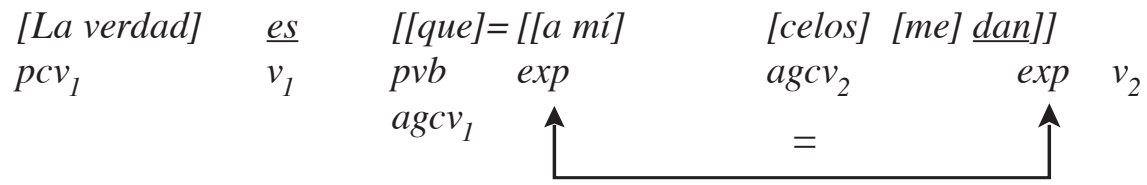




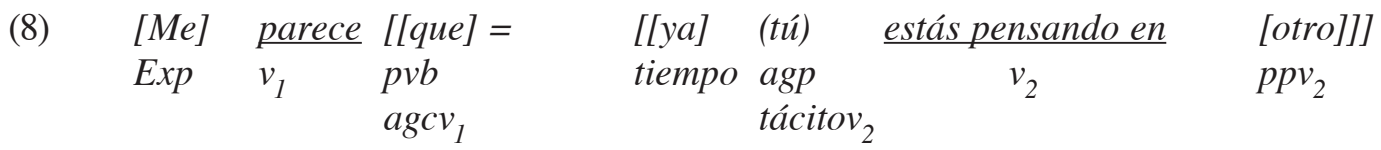

La oración completa para el ejemplo (9) es Las delicias de la vida son las miradas de la diosa, será por eso que el rey se sofoca cuando hay otro hombre que la mira, donde por eso reproduce la oración Las delicias de la vida son las miradas de la diosa, constituyéndose en razón de será. Que es el agente cosa de será, ello será por eso.

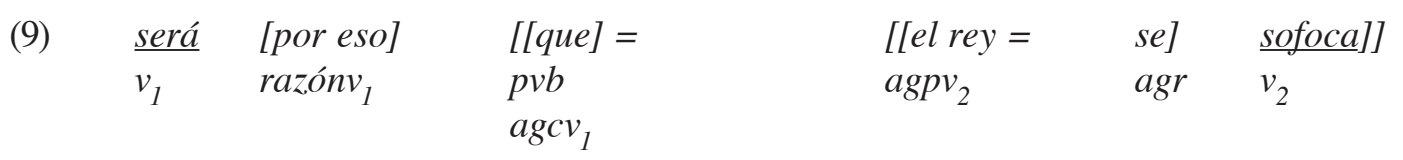

El enlace cuando sería el normativo en el ejemplo (10), lo cual da al constituyente proverbal con que un tinte temporal. Que es el agente cosa de $\boldsymbol{e s}$.

$\begin{array}{lllc}\text { [ahora] } \underline{\text { es }} & {[[q u e]=} & {[(\text { yo })} & \text { voy a bailar }]] \\ \text { tiempo } v_{1} & p v b & \text { ag } & v_{2} \\ & \text { agcv } & \text { tácitov }_{1} & \end{array}$

\subsubsection{Paciente cosa}

El constituyente proverbal con que es paciente cosa del $v_{1}$ en 62 ejemplos. De éstos, 17 se producen con decir, 14 con saber, 4 con haber, y 27 con otros verbos de paciente como pensar, querer, ver, mirar, comprender, creer, esperar, rogar, pedir. Hay cuatro ejemplos que se producen con la interjección verbal ojalá.

(11) $(Y o) \quad[T e]$ estoydiciendo $\quad[[q u e]=[(t u ́)$ cambies $\quad$ [la cara de seriedad] $\begin{array}{lllllll}a g & p p v_{1} & v_{1} & p v b & a g & v_{2} & p c v_{2} \\ \text { tácito } & & & p c v_{1} & \text { tácitov } & & \end{array}$

$\begin{array}{llllcl}\text { (Tú) } & \underline{\text { sabes }} & {[[q u e]=} & {[(y o)} & \text { sigo esperándo } & \text { [te]]] } \\ \text { ag } & v_{1} & p v b & a g & v_{2} & p p v_{2} \\ \text { tácito } & & p c v_{1} & \text { tácitov } & & \end{array}$

(13) Yo pensaba $[[q u e]=[(e l l a) \quad[m e]$ iba a dar $\quad$ [un nietecito con los ojos rubios]]] $\begin{array}{lllllll}a g & v_{1} & p v b & a g & p p v_{2} & v_{2} & p c v_{2}\end{array}$ tácito $\quad$ pcv $_{1} \quad$ tácitov $_{2}$ 


\subsubsection{Modal}

El constituyente proverbal con que es modal del $v_{1}$ en 2 ejemplos.

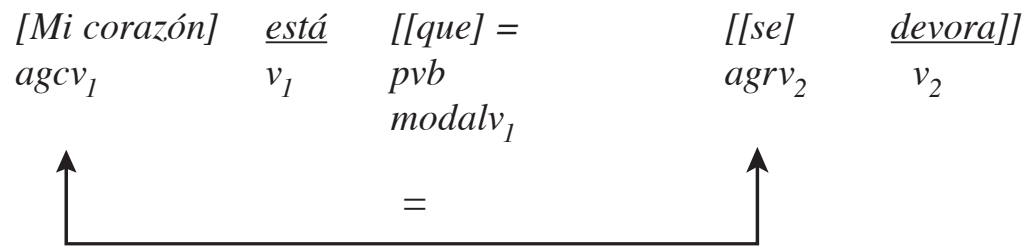

\subsection{Que 'proverbo', verbo $+\left[[(\right.$ preposición $)$ que $\left.]=\left[v_{2}\right]\right]$}

Tabla 3. Que 'proverbo', verbo ${ }_{1}+\left[[(\right.$ preposición $)$ que $\left.]=\left[\mathbf{v}_{2}\right]\right]$

\begin{tabular}{lccc}
\hline Caso del verbo & Preposición + que & $\begin{array}{c}\boldsymbol{\emptyset} \text { preposición }+ \\
\text { que }\end{array}$ & Total \\
\hline Tiempo & 3 & 30 & 3 \\
Razón (por) & 11 & 1 & 41 \\
Finalidad (para) & 20 & 31 & 21 \\
\hline \multicolumn{1}{c}{ Total } & 34 & 65 \\
\hline
\end{tabular}

También consideramos que un 'proverbo' cuando va precedido por una preposición, que introduce un caso del $\mathrm{v}_{1}$. Encontramos 65 ejemplos de este uso de que. Las preposiciones más comunes son por y para, que introducen los casos razón y finalidad del $\mathrm{v}_{1}$. En los tres ejemplos del caso tiempo se usan desde(1) y hasta (2). La preposición antecede a que tal como antecede a cualquier nombre o pronombre. Es de notar que por es elidido en 30 ejemplos y para en 1 .

\subsubsection{Tiempo}

El constituyente proverbal $\left[\left(\right.\right.$ prep) que $=\mathrm{v}_{2}$ ] es tiempo del $\mathrm{v}_{1}$ en 3 ejemplos.

$$
\begin{aligned}
& \text { (Yo) vivo recordando [tu imagen pura] [[desde que] }=[(t u ́) \text { [me] dejaste [solito]]] } \\
& \begin{array}{llllllll}
a g & v_{1} & p c v_{1} & \text { prep pvb } & \text { ag } & p p v_{2} & v_{2} & \text { modal }_{2}
\end{array} \\
& \text { tácito } \\
& \text { tiempov } \text { tácito }_{1}
\end{aligned}
$$




\subsubsection{Razón}

El constituyente proverbal [(por) que e $\mathrm{v}_{2}$ ] es siempre razón del $\mathrm{v}_{1}$, con 41 ejemplos. En 11 de ellos por aparece, pero en 30 de ellos está elidido. En 25 de los casos en que por está elidido, el $\mathrm{v}_{1}$ está en el modo imperativo afirmativo o negativo. En los 5 restantes, que no tiene antecedente verbal, pero este se sobrentiende como imperativo: (Dame) Una piedra que me voy a machacar

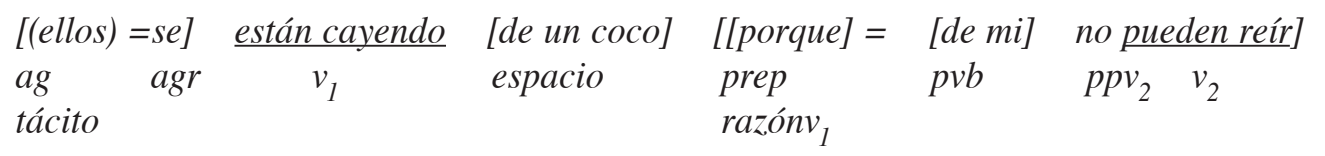

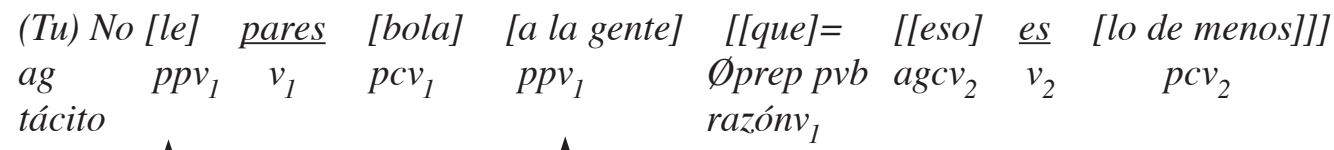

\subsubsection{Finalidad}

El constituyente proverbal [(para) que $=\mathrm{v}_{2}$ ] es siempre finalidad del $\mathrm{v}_{1}$, con 21 ejemplos. En el ejemplo (19) para está elidido.

$\begin{array}{llllll}\text { Aqui } & \text { estoy } & {[y o]} & \text { [1pa'que] } & \text { [tú] } & \text { decidas }] \\ \text { Espacio } & v_{1} & \begin{array}{l}\text { agp } \\ \text { finalidad } v_{1}\end{array} & \text { prep.pvb } & \text { agp } & v_{2}\end{array}$

(19) (Tú) [Le] pones [ungran arrozcolado] [[que] = coma [el rey considerado] $\begin{array}{llllllll}\text { ag } & p p v_{1} & v_{1} & p c v_{1} & \emptyset p r e p & p v b & v_{2} & a g p v_{2}\end{array}$ tácito finalidad $v_{1}$

Si sumamos los usos de que como proverbo en las Tablas 2 y 3 tenemos un total de 150 usos de $\mathrm{v}_{1}\left[\left[(\right.\right.$ prep) que $\left.]=\left[\mathrm{v}_{2}\right]\right]$.

\subsection{Que pronombre relativo, verbo ${ }_{1}\left[[\right.$ nombre $\left.]=[q u e] v_{2}\right]$}

Tabla 4. Que pronombre relativo, verbo $_{1}\left[[\right.$ nombre $\left.]=[q u e] \mathbf{v}_{2}\right]$

\begin{tabular}{|c|c|}
\hline $\begin{array}{c}\text { Nombre } \\
\text { Caso del verbo } 1\end{array}$ & $\begin{array}{c}\text { Que } \\
\text { Caso del verbo }_{2}\end{array}$ \\
\hline $\begin{array}{c}\text { Agente persona } \\
13\end{array}$ & $\begin{array}{c}\text { Agente persona } \\
13\end{array}$ \\
\hline
\end{tabular}


Tabla 4. $Q u e$ pronombre relativo, verbo $_{1}\left[[\right.$ nombre $\left.]=[q u e] \mathbf{v}_{2}\right]$

\begin{tabular}{|c|c|}
\hline $\begin{array}{c}\text { Nombre } \\
\text { Caso del verbo }_{1}\end{array}$ & $\begin{array}{c}\text { Que } \\
\text { Caso del verbo } 2\end{array}$ \\
\hline $\begin{array}{c}\text { Agente cosa } \\
11\end{array}$ & $\begin{array}{c}\text { Agente cosa } \\
4 \\
\text { Paciente cosa } \\
7\end{array}$ \\
\hline $\begin{array}{c}\text { Experimentador } \\
1\end{array}$ & $\begin{array}{c}\text { Experimentador } \\
1\end{array}$ \\
\hline $\begin{array}{l}\text { Paciente persona } \\
15\end{array}$ & $\begin{array}{c}\text { Paciente persona } \\
3 \\
\text { Agente persona } \\
12\end{array}$ \\
\hline $\begin{array}{l}\text { Paciente cosa } \\
64\end{array}$ & $\begin{array}{c}\text { Paciente cosa } \\
25 \\
\text { Agente persona } \\
1 \\
\text { Agente cosa } \\
35 \\
\text { Paciente persona } \\
1 \\
\text { Modal } \\
2\end{array}$ \\
\hline $\begin{array}{c}\text { Tiempo } \\
5\end{array}$ & $\begin{array}{c}\text { Tiempo } \\
5\end{array}$ \\
\hline $\begin{array}{c}\text { Espacio } \\
6\end{array}$ & $\begin{array}{c}\text { Espacio } \\
3 \\
\text { Agente cosa } \\
2 \\
\text { Paciente cosa } \\
1\end{array}$ \\
\hline $\begin{array}{c}\text { Compañía } \\
1\end{array}$ & $\begin{array}{c}\text { Paciente persona } \\
1\end{array}$ \\
\hline $\begin{array}{c}\text { Instrumento } \\
1\end{array}$ & $\begin{array}{c}\text { Modal } \\
1\end{array}$ \\
\hline $\begin{array}{l}\text { Relativo de una } \\
\text { cláusula relativa }\end{array}$ & 2 \\
\hline Sin antecedente verbal & 7 \\
\hline Total & 126 \\
\hline
\end{tabular}




\subsubsection{Paciente cosa}

De los 64 ejemplos en que el nombre es el paciente cosa del $v_{1}$, en 25 que es también el paciente cosa del $\mathrm{v}_{2}$, en 35 que es el agente cosa del $\mathrm{v}_{2}$, en 2 que es el modal del $\mathrm{v}_{2} \mathrm{y}$ en sendos ejemplos que es el agente persona y paciente persona del $\mathrm{v}_{2}$.

\begin{tabular}{|c|c|c|c|c|c|c|}
\hline $\begin{array}{l}Y o) \\
4 g \\
\text { ácito }\end{array}$ & $\frac{\text { Voy a matar }}{v_{1}}$ & $\begin{array}{c}{[[\text { un capricho }]=} \\
p c v_{1}\end{array}$ & $\begin{array}{l}\text { [que] } \\
\text { prr } \\
\text { pcv }\end{array}$ & $\begin{array}{l}(y o) \\
\text { ag } \\
\text { tácito }\end{array}$ & $\frac{\text { tengc }}{v_{2}}$ & $\begin{array}{l}\text { [en el corazón]] } \\
\text { espacio }\end{array}$ \\
\hline
\end{tabular}

(26) (Yo) salía comprar [te] [[una cadena] = [que] [te] llegue [a la rodilla]] $\begin{array}{llllllll}A g & v_{1} & p p v_{1} & p c v_{1} & \text { prr } & p p v_{2} & v_{2} & \text { espacio }\end{array}$ tácito $\operatorname{agcv} v_{2}$

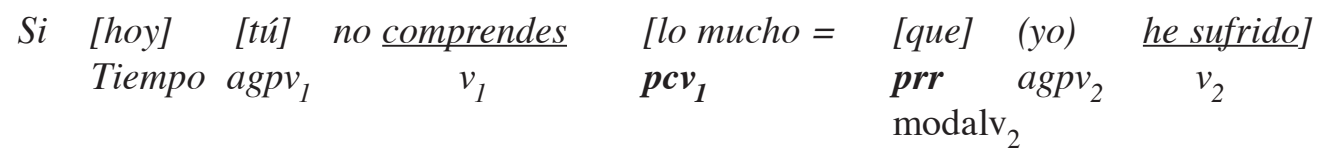

(28) (Yo) Ya no sería [[el mismo soñador] = [que] [a tantos desengaños] [se] enfrentó $\begin{array}{lllllll}\text { Ag tiempo } & v_{1} & \boldsymbol{p c v _ { 1 }} & \boldsymbol{p r r} & p c v_{2} & \operatorname{agrv}_{2} & v_{2} \\ \text { tácito } & & & \boldsymbol{a g p v}_{\mathbf{2}} & & \end{array}$

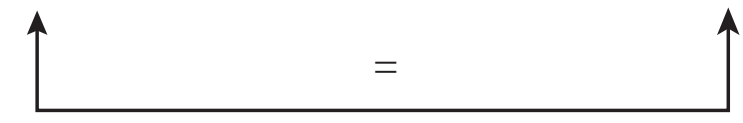

\begin{tabular}{|c|c|c|c|c|c|}
\hline$(Y o)$ & $\underline{s O Y}$ & {$[$ el cantante $]=$} & [que] & [hoy] & han venido a escuchar \\
\hline $\begin{array}{l}A g \\
\text { tácito }\end{array}$ & $v_{l}$ & $p c v_{1}$ & & tiempo & $v_{2}$ \\
\hline
\end{tabular}

\subsubsection{Tiempo}

En los 5 ejemplos en que el nombre es el tiempo del $\mathrm{v}_{1}$, que es también el tiempo del $\mathrm{v}_{2}$.

$\begin{array}{ll}{[\text { Siempre }]=} & \text { [que }] \quad \text { empieza a llover }] \\ \text { tiempov }_{1} & \begin{array}{l}\text { prr } \\ \text { tiempov }_{2}\end{array}\end{array}$

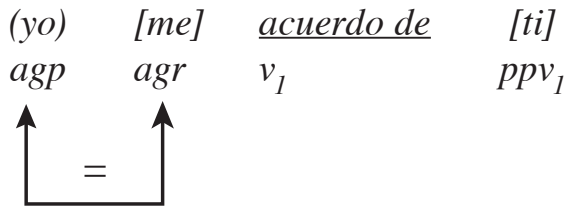

\subsubsection{Espacio}

De los 6 ejemplos en que el nombre es el espacio del $\mathrm{v}_{1}$, en 3 que es también el espacio del $\mathrm{v}_{2}$, en 2 que es el agente cosa del $\mathrm{v}_{2} \mathrm{y}$ en 1 que es el paciente cosa del $\mathrm{v}_{2}$. 
(31)

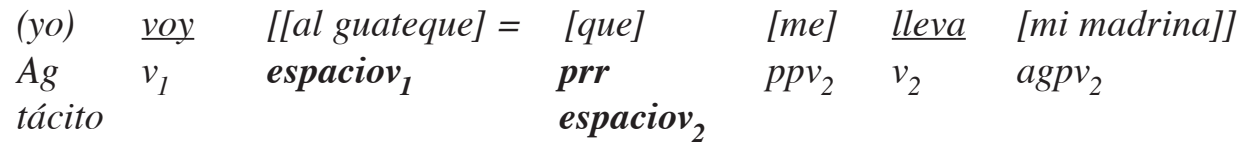

(Tú) Mónta [te] [en tu alfombra],

$$
\begin{aligned}
& \text { [[esa] }=\text { [que] } \underline{\text { es }} \text { [voladora }] \\
& \begin{array}{llll}
\operatorname{agcv}_{2} & \boldsymbol{p p r} & v_{2} & \text { modalv }_{2} \\
\boldsymbol{a g c v _ { 2 }} & &
\end{array}
\end{aligned}
$$$$
\text { Ag } \quad v_{1} \quad p_{1} v_{1} \text { espaciov }_{1}
$$

Tácito

(33) (Yo) Estoyahogándo [me] [[entre cosas y...] = [que] no puedo comprender]

$\begin{array}{llllll}A g & v_{1} & \text { agrv }_{1} & \text { espaciov }_{1} & \text { ppr } & v_{2}\end{array}$
tácito

\subsubsection{Instrumento}

Hay un ejemplo en que el nombre es instrumento del $\mathrm{v}_{1} \mathrm{y}$ que es modal del $\mathrm{v}_{2}$.

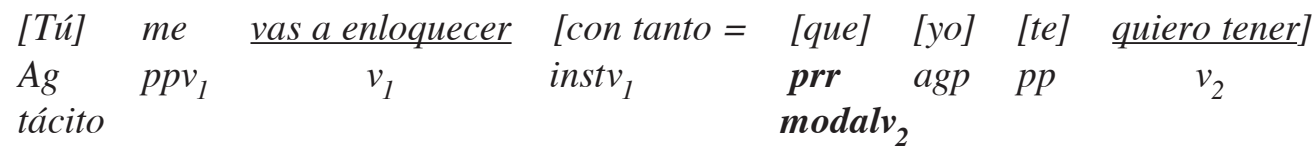

\subsubsection{Compañia}

Hay un ejemplo en que el nombre es compañía del $v_{1}$ mientras que es paciente persona del $\mathrm{v}_{2}$. Es interesante ver como que es relativo del término de preposición mis padres, más que del constituyente completo [del brazo de mis padres].

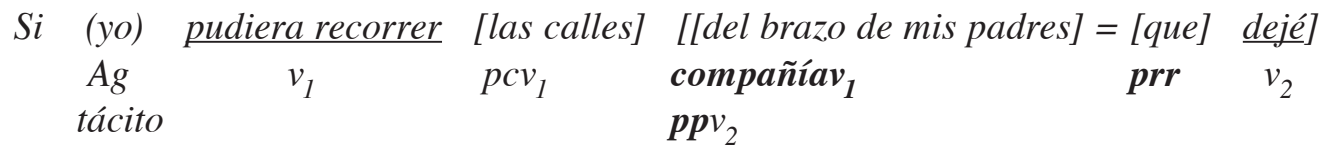

4.3.9. Que relativo de una cláusula relativa

Encontramos solo dos ejemplos de este interesante uso de que. Que es agente del $v_{1}$ y dentro de la cláusula, agente del $\mathrm{v}_{2}$.

(36) [El que tenga estas tres cosas] $=$ [que] [le] dé [gracias] [a dios]

[El que tenga un amor] $=$ [que] [lo] $\underline{\text { cuide }}$

\subsubsection{Sin antecedente verbal}

Hay 7 ejemplos en los cuales que es pronombre relativo sin antecedente verbal. 
(38)

$\left[[\right.$ Miel $]=\left[\begin{array}{llll}{[q u e]} & (t u ́) & {[m e]} & \underline{d a s} \\ p c v & a g p v & p p v & v\end{array}\right.$

4.3.11. Distribución de relaciones de caso para el nombre antecedente y que

Tabla 5. Distribución de las relaciones de caso del nombre antecedente con el verbo 1 y de que con el verbo

\begin{tabular}{cc}
\hline Nombre & Que \\
Caso del verbo $_{1}$ & Caso del verbo $_{2}$ \\
\hline
\end{tabular}

\begin{tabular}{|c|c|c|}
\hline Agente persona & 13 & 26 \\
\hline Agente cosa & 11 & 41 \\
\hline Experimentador & 1 & 1 \\
\hline Paciente persona & 15 & 5 \\
\hline Paciente cosa & 64 & 33 \\
\hline Tiempo & 5 & 5 \\
\hline Espacio & 6 & 3 \\
\hline Compañía & 1 & - \\
\hline Instrumento & 1 & - \\
\hline Modal & - & 3 \\
\hline Total & 117 & 117 \\
\hline
\end{tabular}

La Tabla 5 muestra la diferente distribución de relaciones de caso entre el nombre antecedente y el $\mathrm{v}_{1}$, y el pronombre relativo que y el $\mathrm{v}_{2}$. Las relaciones más comunes del nombre antecedente de que con el $\mathrm{v}_{1}$ son: paciente cosa (51 por ciento), paciente persona (12 por ciento), agente persona (10 por ciento) y agente cosa (9 por ciento). Las relaciones más comunes de que con el $\mathrm{v}_{2}$ son agente cosa (33 por ciento), paciente cosa (27 por ciento) y agente persona (21 por ciento).

El nombre aparece en la relación de caso agente persona en 13 ejemplos, que, en 26 ejemplos, o 2:1. El nombre aparece en la relación de caso agente cosa en 11 ejemplos, que, en 41 
ejemplos o 1:4. No hay variación entre el nombre y que en el caso experimentador. El nombre aparece en la relación de caso paciente persona en 15 ejemplos, que, en 5 ejemplos o 3:1. El nombre aparece en la relación de caso paciente cosa en 64 ejemplos, que, en 33 ejemplos o 2:3. No hay variación entre el nombre y que en el caso tiempo. El nombre aparece en la relación de caso espacio en 6 ejemplos, que, en 3 ejemplos o 2:1. El nombre aparece en la relación de caso compañía $1 \mathrm{vez}$, que 0 . El nombre aparece en la relación de caso instrumento $1 \mathrm{vez}$, que, 0. Que aparece en la relación de caso modal 3 veces, el nombre 0.

Estos números muestran como el nombre pronominalizado por que y el nombre que son independientes en su relación con los respectivos verbo $\mathrm{y}_{1}$ verbo $_{2}$, encontrándose la variación más alta en el caso agente cosa.

Cortez-Rodríguez, en "El que relativo y su antecedente en la lengua hablada" (1987: 308), encuentra una distribución similar de funciones desempeñadas por que en su proposición, salvando las diferencias en nomenclatura: Sujeto (56.4), complemento directo (24.5), complemento indirecto (1.2), complemento circunstancial (16.9).

Así, completamos el análisis de los dos usos más comunes de que: proverbo y pronombre relativo. A continuación, estudiamos otros usos de que encontrados en el material, usando la gramática verbal.

\subsection{Qué pronombre interrogativo}

En la oración interrogativa, qué pregunta por un constituyente caso del verbo. Dividimos los ejemplos para representar oraciones interrogativas directas e indirectas porque funcionan de manera diferente. En la interrogativa directa, qué constituye relación solo con el verbo principal. En la interrogativa indirecta, qué se relaciona con el $\mathrm{v}_{1} \mathrm{y}$ también con el $\mathrm{v}_{2}$. ¡Lo que nos faltaba! A ver.

\subsubsection{Interrogativa directa: [(prep) qué (nombre)] $v_{1}$}

Tabla 6. Qué pronombre interrogativo directo

\section{Caso del verbo}

\begin{tabular}{lc}
\hline Agente cosa & 1 \\
Paciente cosa & 2 \\
Razón & 5 \\
\hline Total & 8 \\
\hline
\end{tabular}


Encontramos 8 ejemplos de interrogativa directa. En estos, qué, pronombre interrogativo, constituye relación con el verbo como agente en un ejemplo, paciente cosa en 2 ejemplos y razón en 5 ejemplos. En 7 de los ejemplos hay solo un verbo. En el ejemplo 39 hay dos.

$\begin{array}{lllll}\text { ¿[Qué] } & \underline{e s} & {[[l o]=} & \text { [que] } & \text { pasa]? } \\ \text { pi } & v_{1} & p c v_{1} & \text { prr } & v_{2} \\ \text { agcv }_{1} & & & \text { agcv } & \end{array}$

En el ejemplo 40, el qué interrogativo tiene carácter adjetivo, al referirse concretamente a un nombre:

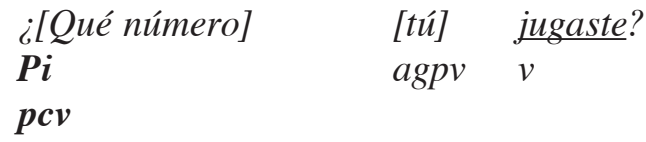

$\begin{array}{lllcl}\text { ¿[Por } & \text { qué] } & \text { (tú) } & \text { no has vuelto a pasar } & \text { [por mi casa]? } \\ \begin{array}{l}\text { Prep } \\ \text { razón }\end{array} & \text { ag } & \text { tácito } & v & \text { espacio } \\ \text { tazón } & & & \end{array}$

4.2.2. Interrogativa indirecta: $v_{1}\left[\left(\right.\right.$ prep) qué (nombre) $\left.v_{2}\right]$

Tabla 7. Qué pronombre interrogativo indirecto

\begin{tabular}{cc}
\hline Caso del verbo & Caso del verbo \\
& \\
\hline Agente cosa & Agente cosa \\
& 3 \\
Paciente cosa & Paciente cosa \\
7 & 3 \\
Razón & Razón \\
1 & 2 \\
& \\
\hline Total & Total \\
8 & 8 \\
\hline
\end{tabular}

En 7 de los 8 ejemplos de interrogativa indirecta, qué pronombre interrogativo, es paciente cosa del $\mathrm{v}_{1}$; en uno qué es razón. Al mismo tiempo que establece relación con el $\mathrm{v}_{1}$, qué establece relación con el $\mathrm{v}_{2}$ : en 3 ejemplos como paciente cosa, en 3 como agente cosa, y en 2 como razón. Esta doble relación de qué con el verbo y el verbo $_{2}$ solo sucede con el pronombre interrogativo en la pregunta indirecta. 
(42)

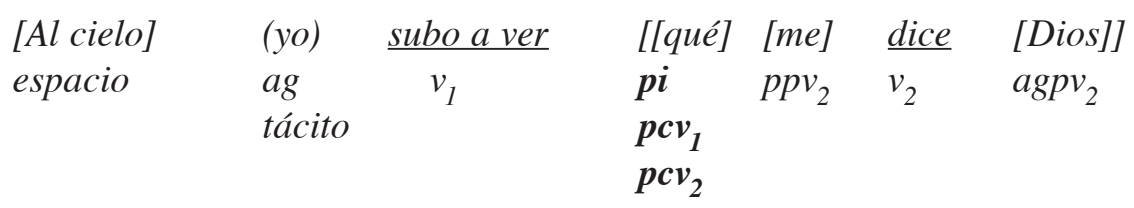

$$
\begin{aligned}
& \text { (Tú) Di [me] [[qué] [te] estápasando] } \\
& \begin{array}{llllll}
a g & v_{1} & p p v_{1} & p i & \exp v_{2} & v_{2}
\end{array} \\
& \text { tácito } \quad \text { pcv } \\
& \operatorname{agcv}_{2}
\end{aligned}
$$

En (44) qué antecedido de la preposición por es razón del $\mathrm{v}_{1} \mathrm{y}$ del $\mathrm{v}_{2}$.

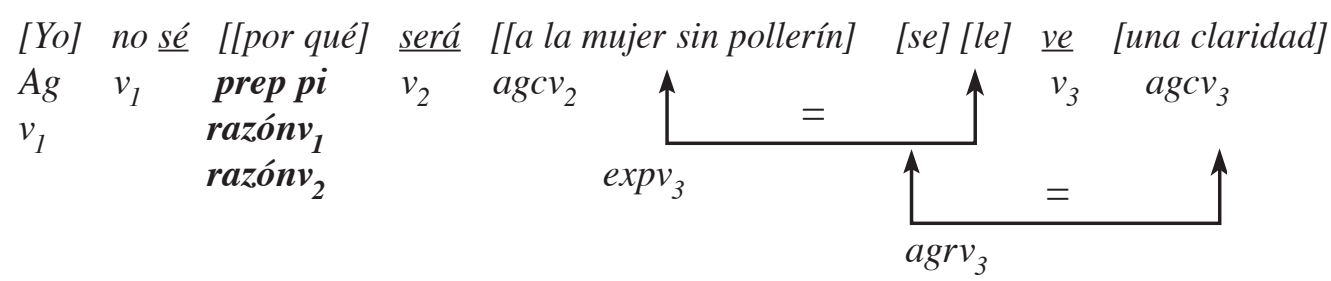

Tal vez esta doble relación del pronombre interrogativo indirecto qué con el verbo ${ }_{1}$ y el verbo ${ }_{2}$ permita que en español podamos hacer la doble pregunta, ¿Que qué?, donde un que reproduce al verbo y el otro a un constituyente del verbo ${ }_{2}$.

\subsubsection{Que pragmático, Øverbo ${ }_{1}$ [que]}

Tabla 8. Que pragmático, Øverbo 1 [que]

\begin{tabular}{ccccc}
\hline Clase & $\begin{array}{c}\text { Qué exclamativo } \\
\text { (Decir) } \\
\emptyset_{1}+[\text { qué nombre }]\end{array}$ & $\begin{array}{c}\text { Que declarativo } \\
(\text { Decir })\end{array}$ & $\begin{array}{c}\text { Que desiderativo } \\
(\text { Desear }) \\
\mathrm{v}_{1}+\left[\text { que }=\mathrm{v}_{2}\right]\end{array}$ & Total \\
\hline Total & 18 & 10 & 11 & 39 \\
\hline
\end{tabular}

\section{5. $<Q u e>$ conjunción $\mathrm{v}_{1}<q u e>\mathrm{v}_{1}$, conjunción verbal [nombre <que $>$ nombre], conjunción nominal}

Que también es usado como conjunción uniendo dos verbos en el nivel sintáctico o dos nombres en el nivel morfológico. Expresamos esta categoría de El que pragmático se caracteriza porque el verbo ${ }_{1}$ no está expresado, aun cuando se puede colegir. Generalmente corresponde a decir en las oraciones exclamativas y declarativas y, como su nombre lo indica, a desear en las desiderativas. 


\subsubsection{Qué exclamativo}

Encontramos 18 ejemplos de qué exclamativo. El qué exclamativo tiene función adjetiva o adverbial según enfatice el concepto expresado por el nombre sustantivo o el nombre adjetivo. Antecede a un nombre sustantivo o nombre adjetivo, con modificadores o no y con verbo o sin él. ¡Qué cabeza!, ¡Qué rica mamita!, ¿Qué bueno baila Celina!

\subsubsection{Que declarativo}

Encontramos 10 ejemplos de que declarativo. En ellos que es 'proverbo' porque reproduce al $\mathrm{v}_{2}$. Ay, que mira, Que la voy a agarrar.

\subsubsection{Que desiderativo}

Encontramos 11 ejemplos de que desiderativo. En ellos que es 'proverbo' porque reproduce al $v_{2}$. En todos los ejemplos el verbo está en el modo subjuntivo. Que sea tu alma mi alma, Que no se apague la llama de amor.

que a través de $<>$.

\subsection{Que conjunción verbal}

El uso de que como conjunción verbal se caracteriza porque los dos verbos unidos por la conjunción están al mismo nivel. Encontramos 3 ejemplos de que conjunción copulativa, uno de aunque conjunción adversativa, y 4 de que conjunción ilativa.

Como conjunción copulativa, que enlaza dos verbos al mismo nivel, denotando simultaneidad de las acciones.

(45) $[$ Cuando $=$ [el rey] llega [de tarde] <que> mira [el jardín florecido]] [se] pone [más engreído] $\begin{array}{lllllllll}p v b & a g v_{2} & v_{2 a} & \text { tiempov }_{2} & v_{2 b} & p c v_{2 b} & \text { agr } & v_{1} & \text { modal } v_{1}\end{array}$ tiempov $_{2}$

$$
\begin{array}{cc}
\text { Chupa <que> chupa } \\
v_{l a} & v_{l b}
\end{array}
$$

$\begin{array}{llllll}(Y o) & {[L o]} & \text { oigo gritar }<q u e> & (e ́ l) & \text { estállamando } & \text { [a madrina] } \\ \text { agp } & p p v_{1} & v_{1 a} & \text { agp } & v_{1 b} & p p v_{1 b} \\ \text { tácito } & & & \text { tácito } & & \end{array}$

Como la conjunción adversativa, aunque, que enlaza dos verbos al mismo nivel, denotando cierta oposición entre las acciones.

$$
\begin{aligned}
& \text { Aun<que }>[(y o)=\text { me esté muriendo } \quad \text { [por tenerla] no olvide } \quad[[q u e]=\underline{\text { hay }} \text { [un hijo] }
\end{aligned}
$$

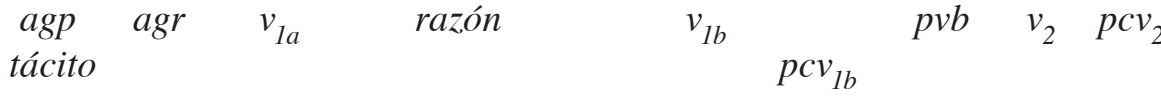

Como conjunción ilativa, que introduce el caso ‘consecuencia' de la acción o acciones expresadas en la oración antecedente. Encontramos los siguientes ejemplos. 
(49) Si hoy tú no comprendes que siempre te amaré que venga dios del cielo y te diga

(50) Qué es lo que pasa que nos estamos alejando tanto.

(51) Me dio una fiebre que fui a parar a enfermería.

(52) Fue tan buena la nota que dio aquel humilde trompeta que entre acordes de cariño eterno se fue ella con él.

\subsubsection{Que conjunción nominal}

En los 4 ejemplos de uso de que como conjunción nominal, que es parte del constituyente modal en 3 y del constituyente paciente cosa en uno. En todos ellos que tiene carácter comparativo.

$$
\begin{aligned}
& \text { [Lucero espiritual] eres [más alto } \leq q u e>\text { el hombre] } \\
& \text { agc } v \quad \text { modal (cantidad) } \\
& \begin{array}{lllll}
\text { me he convertido en } & \text { [una cosa }= & \text { [que] no hace } & \text { [otra cosa más } \leq q u e>\text { amarte] }] \\
\text { agr } v_{1} & p c v_{1} & \begin{array}{l}
\text { prr } \\
\text { agcv }
\end{array} & v_{2} & p c v_{2}
\end{array}
\end{aligned}
$$

\subsection{Lo que, el que, aquel que}

Este que parece ser siempre pronombre relativo nunca proverbo, porque lo, el y aquel actúan como núcleos del constituyente nominal y antecedentes de que y constituyen relación con el $\mathrm{v}_{1}$, mientras que se relaciona con el $\mathrm{v}_{2}$. Concordamos con Alonso (1991: 325), quien señala que el artículo actúa como transpositor a categoría sustantiva.

Es importante mencionar que en el material, en dos ejemplos lo es artículo:

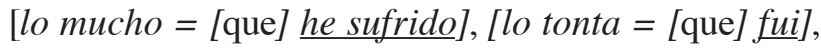

donde mucho, y tonta actúan como núcleos del constituyente.

El que se presenta en 4 ejemplos, uno de los cuales uno es al que. Aquel que se presenta en un ejemplo. Lo que se presenta en 18 ejemplos, constituyéndose en el más común. Que constituye relación con el $\mathrm{v}_{2}$ como agente persona (4 ejemplos), agente cosa (9), y paciente cosa (10). Ejemplos selectos siguen:

$\begin{array}{lllll}\text { [Aquel }= & \text { [que] } & \text { guarda]] } & \text { [siempre] } & \text { tiene } \\ \text { Agpv }_{1} & \text { prr } & v_{2} & \text { tiempo } v_{1} & v_{1} \\ & \text { agpv }_{2} & & & \end{array}$

$\begin{array}{llllll}{[[\text { Lo] }=} & {[\text { que }]} & {[m e]} & \underline{\text { toca }]} & \underline{\text { es }} & \text { sufrir } \\ \operatorname{agcv}_{1} & \text { prr } & \exp & \mathrm{v}_{2} & \mathrm{v}_{1} & \operatorname{agcv}_{1} \\ & \operatorname{agcv}_{2} & & & & \end{array}$




\begin{tabular}{|c|c|c|c|c|c|c|c|}
\hline (Tú) No & [me] & escuchas & {$[[l o]$} & [que] & (yo) & [te] & \\
\hline $\begin{array}{l}\text { agp } \\
\text { tácito }\end{array}$ & $p p v_{1}$ & $v_{1}$ & $p c v_{1}$ & $\begin{array}{l}p r r \\
p c v_{2}\end{array}$ & $a g p$ & $p p v_{2}$ & $v_{2}$ \\
\hline
\end{tabular}

Por otro lado, todo actúa como adjetivo en [todo lo $=$ [que] he sido] y [todo lo $=$ que fue el tiempo lo dejó atrás]. En Tu amor no se parece a nada de lo que fui buscando, aunque a primera vista a nada de lo que fui buscando parezca un solo constituyente, hay en realidad dos pacientes cosa: uno en relación de objeto directo y otro en relación de objeto indirecto con el $\mathrm{v}_{1}$ [nada] [de lo = [que] fui buscando]. Aquí sí viene al caso la explicación tradicional.

$$
\begin{aligned}
& \text { [Tu amorno se] parece [a nada] [de lo = [que] (yo) fuibuscando] } \\
& \begin{array}{llllllll}
a g c v_{1} & a g r & v_{1} & p c-o d & p c-o i & p c v_{2} & a g & v_{2}
\end{array}
\end{aligned}
$$

\section{Conclusiones y recomendaciones}

Los 343 ejemplos de usos de que encontrados en el material fueron susceptibles de análisis y explicación usando el marco teórico propuesto, respetando los principios hjelslevianos de coherencia y simplicidad. La capacidad explicativa de la gramática verbal se manifiesta en la manera elegante como explica misterios remotos y nunca bien nombrados del que.

La disputa, que comenzó con Bello y Cuervo en los 1860 y a la que todavía alude Alonso (1991), había permanecido sin resolver:

Según nos dice Bello, en estas oraciones Que la tierra se mueve alrededor del sol es cosa averiguada, Los animales se diferencian de las plantas en que sienten y se mueven, que es un sustantivo equivalente a esto y perteneciente a la proposición principal. Esta explicación me parece demasiado artificial, y ofrece las dificultades siguientes que pueden pasar por argumentos a favor del carácter relativo de este vocablo, del cual lo despojaríamos haciéndole pertenecer a la preposición subordinante...Estos son los hechos que señalan el camino recorrido por quod para llegar al oficio de anunciativo, en que lo ha reemplazado que, y al cual no conviene ninguna denominación de la nomenclatura conocida (Bello y Cuervo 1874: 441). Nota 58 (num 316).

Algunos autores, basándose en la posible omisión de la preposición, antes (de) que vengas, piensan que el transpositor que es de naturaleza relativa. Pero creemos que tal hipótesis es difícilmente sostenible, pues no sabríamos decir qué papel cumple el $q u e^{2}$ en la estructura transpuesta, ni cual podría ser su 'antecedente': antes de que tu llegues todo habrá concluido (Que \# antes) (Genaro Alonso 1991: 332).

Aportamos la siguiente resolución, según nuestros datos y análisis, a la discusión anotada arriba:

- $\quad$ En la estructura $\mathrm{v}_{1}\left[\left[(\right.\right.$ preposición) que $\left.]=\left[\mathrm{v}_{2}\right]\right]$, que es nombre, como dice Bello, es el núcleo del constituyente, pero no pertenece a la proposición principal, como recalca Cuervo. Pertenece al constituyente proverbal.

- $\quad$ En el ejemplo de Alonso, el papel que cumple el que, es el de 'proverbar' el constituyente tiempo a través del 'proverbo': [[antes de que] = [[tú] llegues] ], morfológicamente, $\left[[\right.$ nombre adverbio-(preposición)- proverbo $\left.]=\left[\mathrm{verbo}_{2}\right]\right]$, un constituyente común en español, donde dos nombres van unidos por preposición. Aquí que no tiene antecedente: en su función de 'proverbo' lo es. Si elidimos la preposición [[antes que] = [tú] llegues], se produce un cambio morfológico en antes, que pasa de ser nombre adverbio a ser simplemente adverbio de que, [[adverbio- proverbo $]=\left[\right.$ verbo $\left.\left._{2}\right]\right]$. 
Es de notar que estructuras del tipo el cuchillo con que corto se perdió, donde que, pronombre relativo, va precedido de preposición, no fueron encontradas en el análisis. Adherimos a la explicación de María Álvarez Martínez (1986: 118) de que en estos casos la preposición no traspone, sino que es índice funcional que señala la relación de caso que el grupo sintágmatico establece con el verbo ${ }_{2}$, en este caso, instrumento de cortar. [El cuchillo con que corto $]=[\mathrm{se}]$ perdió, morfológicamente $\left[[\right.$ nombre $]<$ prep $>[$ que $\left.\left.] \mathrm{v}_{2}\right]\right]$.

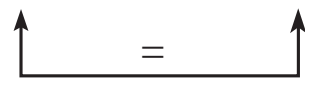

En resumen:

5.1. En el nivel sintáctico, el del verbo ${ }_{1}$, que funciona como:

5.1.1. Enlace entre verbo ${ }_{1}$ y verbo ${ }_{2}$, nombre y 'proverbo', en relación de caso con el $\mathrm{v}_{1}$ y reproduciendo al verbo ${ }_{2}$, sin establecer relación de caso con él, Dice que no tengo remedio, Aquí estoy yo pa' que tú decidas.

5.1.2. Pronombre interrogativo directo, ¿Qué número tú jugaste?

5.1.3. Que pragmático:

- qué adjetivo o adverbio, ¡Qué cabeza!, ¿Qué rica mamita!;

- que proverbo, ay, que mira, que viva alegre la sabana.

5.1.4. Conjunción verbal, Chupa que chupa.

5.2. En el nivel morfológico, el de los constituyentes, que funciona como:

5.2.1. Enlace entre nombre y verbo ${ }_{2}$, pronombre relativo, y nombre en relación de caso con el $\mathrm{v}_{2}$ no con el $\mathrm{v}_{1}$, Eres una fuente que refresca.

5.2.2. Conjunción nominal, Pudo más el amor que el dinero.

5.3. En los niveles morfológico y sintáctico, a la vez, que funciona como:

5.3.1. Pronombre interrogativo indirecto, Hasta el cielo subo yo a ver qué me dice Dios.

Nuestro siguiente paso es investigar la aplicabilidad de este marco teórico a quien, como, cuando y donde, palabras que como que se caracterizan por cumplir diversas funciones a un mismo tiempo. 


\section{Bibliografía}

Alarcos Llorac, E. 1973. Estudios de Gramática Funcional del Español. Madrid: Gredos.

Alonso Megido, G. 1991. "Los relativos en español: doble caracterización funcional”. Verba. 18: 323-351.

Álvarez Martínez, M. 1986. “Dos aspectos del funcionamiento del relativo”. REL. 16: 113- 131.

Bello, A. y Rufino José Cuervo. 1964. Gramática de la Lengua Castellana. $7^{\mathrm{a}}$ ed. Buenos Aires: Sopena.

Bloomfield, L. 1933. Language. New York: H. Holt \& Co.

Brown, K. and J. Miller. 1991. Syntax: A Linguistic Introduction to Sentence Structure. 2a ed. Londres: Harper Collins Academic.

Chierchia, G. and S. McConnell-Ginet. 1990. Meaning and Grammmar. Cambridge, MA: MIT.

Contreras, H. 1976. A Theory of Word Order with Special Reference to Spanish. Amsterdam: North Holland.

Contreras, H. (ed.). 1974. Los Fundamentos de la Gramática Transformacional. $3^{\text {a }}$ ed. México, D.F.: Siglo Veintiuno.

Corrales, N. 2001. "Enseñanza de la gramática y cultura hispánicas a través de canción y danza caribes". Cauce, Revista de la Lengua y su Didáctica. 24: 405- 416.

Cortéz-Rodríguez, L. 1987. "El que relativo y su antecedente en la lengua hablada". REL. 17: 301- 325.

Cuervo, R.J. 1955. Apuntaciones ccríticas sobre el lenguaje bogotano. 9a ed. Bogotá: Instituto Caro y Cuervo.

1981. Notas a la Gramática de la Lengua Castellana de Don Andrés Bello. Bogotá: Instituto Caro y Cuervo.

Dijk, T. A. van. 1972. Some Aspects of Text Grammars. The Hague: Mouton.

Fillmore, C. 1968. "The Case for Case”. Universals in Linguistic Theory En: E. Bach (ed.). New York: Holt, Rinehart and Winston. 
1971. “Some Problems for Case Grammar”. Working Papers in Linguistics. 10: 245-265.

Gil, A. 2000. "La Gramática de Andrés Bello vista desde la Perspectiva de la Gramática del Texto". La gramática de Andrés Bello (1847- 1997). Actas del CongresoHomenaje celebrado con motivo del ciento cincuenta aniversario de la Gramática de la Lengua Castellana destinada al uso de los americanos. Bonn: Romanistischer Verlag. 375- 390.

Gili y Gaya, S. 1980. Curso Superior de Sintaxis Española. 9a ed. Habana: Pueblo y Educación.

Haegeman, L. 1994. Introduction to Government and Binding Theory. $2^{\mathrm{a}}$ ed. Oxford: Blackwell.

Nuessel, F. 1975. “The Spanish Conjunction que”. Lenguaje y Ciencias. 15: 124- 132.

Palmer, R.F. 1994. Gramatical Roles and relations. Cambridge: Cambridge UP.

Pruñonosa-Tomás, M. 1990. "De la cláusula relativa: los relativos dónde y cuándo". Valencia: Artes Gráficas Soler, S.A.

Real Academia Española. 1982. Esbozo de una Nueva Gramática de la Lengua Española. Madrid: Espasa-Calpe.

Rivero, M.L. 1982. "Las relativas restrictivas con que”. Nueva Revista de Filología Hispánica 31: 194- 234.

Wells, R. 1958. "Immediate constituents". Readings in Linguistics. En Martin Joos (ed.). New York: American Council of Learned Societies. 186- 207. 